\title{
Thermotropic polypeptides bearing side-on mesogens
}

\author{
Kathleen E Schaefer, ${ }^{\S}$ Patrick Keller, ${ }^{\dagger}$ Timothy J Deming ${ }^{*}$
}

\section{Supporting Online Information}

\section{Experimental}

Instrumentation. Infrared spectra were recorded on a Perkin-Elmer 1600 FTIR spectrophotometer using $\mathrm{NaCl}$ plates. Circular dichroism spectra were recorded on an OLIS RSM CD spectrophotometer running in conventional scanning mode. NMR spectra were measured on a Bruker AVANCE $200 \mathrm{MHz}$ spectrometer using $\mathrm{CDCl}_{3}$ as solvent. The solvent peak was used as an internal standard. Differential scanning calorimetry (DSC) analyses were performed on a TA Instruments DSC 2920 differential scanning calorimeter. DSC samples were scanned at a heating rate of $10{ }^{\circ} \mathrm{C} / \mathrm{min}$ from 0 to $200{ }^{\circ} \mathrm{C}$ with data collected during the second cycle. Calibrations were made using indium as the standard for both temperature transitions and heats of fusion. Melting transition temperatures were determined as the peak maxima of the transition. Thermogravimetric analyses were recorded on a Metler 851e TG coupled to a Pfeiffer ThermoStar Mass Spectrometer for thermogravimetric analysis with an option for evolved gas analysis. All analyses were from 25 to $600{ }^{\circ} \mathrm{C}$ at a rate of $10{ }^{\circ} \mathrm{C} /$ minute and were performed under a nitrogen atmosphere. Liquid crystalline textures were observed using crossed polarizers on a Nikon Optiphot-100S microscope equipped with an Instec HCS302 heat stage and STC2EA controller. Wide-angle x-ray diffraction patterns were recorded on a custom built spectrometer equipped with a MAR 345mm diameter image plate detector and $18 \mathrm{~kW}$ Rigaku rotating anode

x-ray generator emitting $\mathrm{Cu} K \alpha$ radiation $(\lambda=1.54 \AA)$ with a sample to detector distance of 
$178 \mathrm{~mm}$. X-ray samples were heated in situ using an Instec HCS302 heat stage mounted in the beam path. GPC measurements were performed using a Waters 2695 Separation Module with a 2414 Refractive Index Detector and a 2996 Photodiode Array Detector. Separations were effected using four Waters Styragel columns (HR 0.5, HR 2, HR 4 and HR 5) at $25{ }^{\circ} \mathrm{C}$ and the samples were analyzed in THF eluent with a flow rate of $1 \mathrm{~mL} /$ minute.

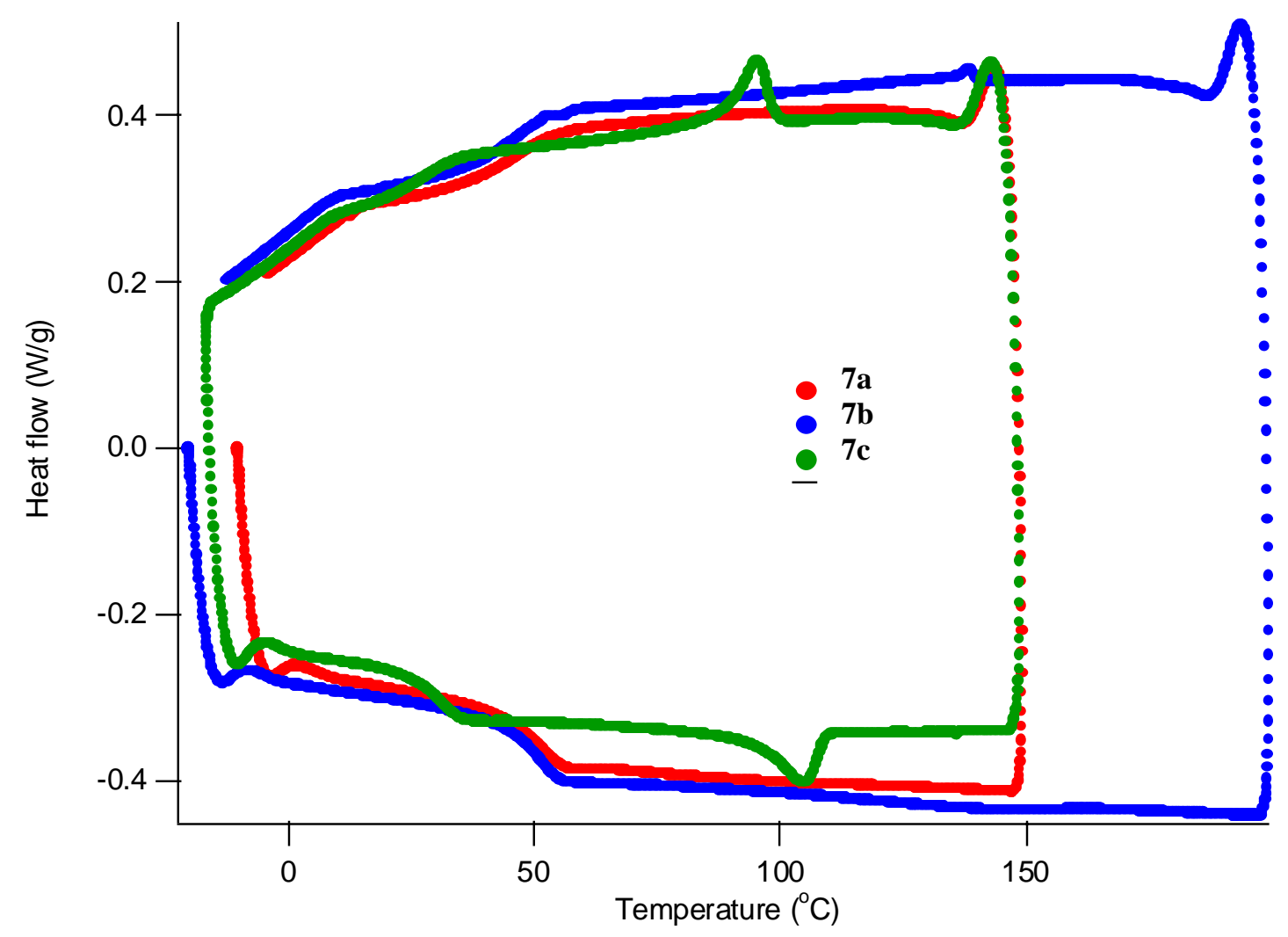

Figure S1 Full DSC data for polypeptides 7a,b,c. 


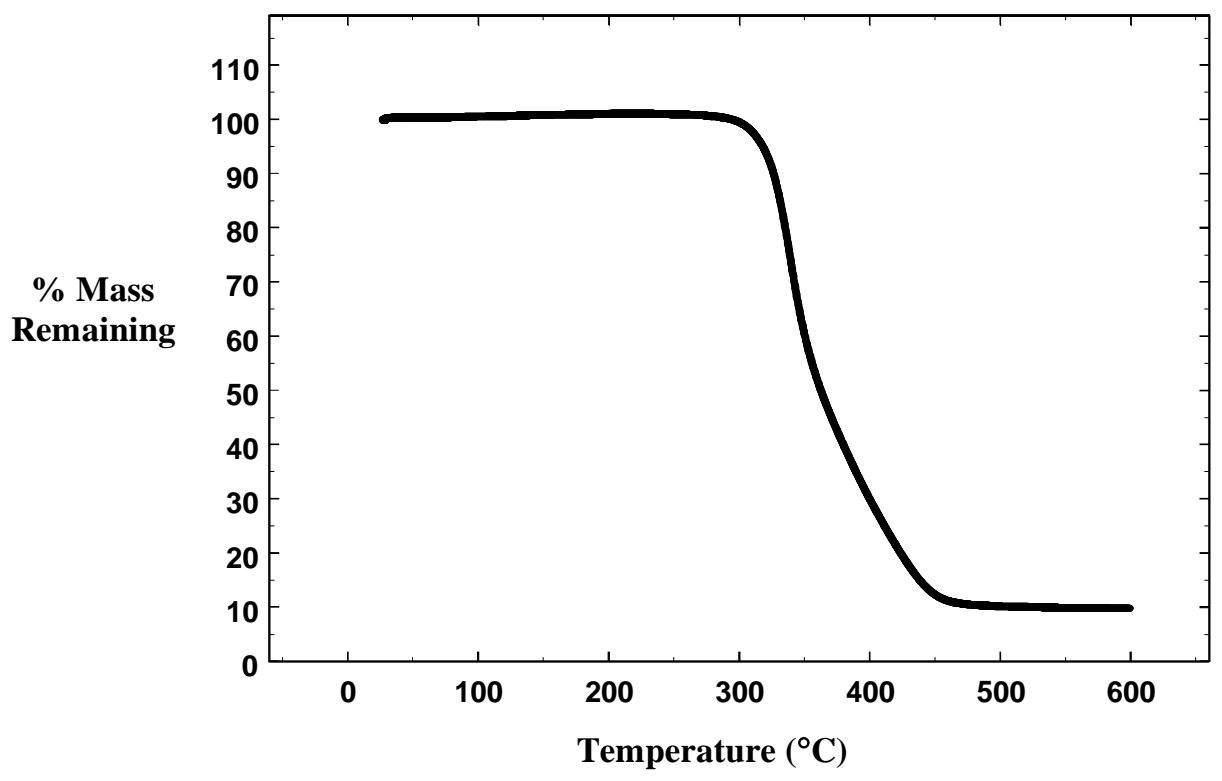

Figure S2 TGA data for polypeptide $\mathbf{7 b}$.

Synthesis. THF, hexane, toluene, ethyl acetate, and $\mathrm{CH}_{2} \mathrm{Cl}_{2}$ were purified by purging with nitrogen followed by passage through columns of activated alumina. Polymerizations were conducted in a nitrogen purged Braun dry box. Synthetic procedures are as follows.

4-(2,5-Di(4'-butyloxybenzoyloxy)benzoyloxy)butyric acid (3a). 3a was prepared according to a literature procedure with the following modifications. Briefly, 2,5dihydroxybenzoic acid was first coupled with 4-bromobutyrate benzyl ester (prepared by DCC/DMAP coupling of 4-bromobutyric acid and benzyl alcohol) to give 4-(2,5dihydroxybenzoyloxy)butyrate benzyl ester (1a). This compound was diesterified with 4butoxybenzoic acid, to yield 4-(2,5-di(4'-butyloxybenzoyloxy)benzoyloxy)butyrate benzyl ester (2a). The benzyl ester was then removed by hydrogenation to give the product, 3a. ${ }^{1} \mathrm{H}$ NMR $\left(\mathrm{CDCl}_{3}\right): \delta 1.00\left(\mathrm{t}, 6 \mathrm{H},-\mathrm{CH}_{3}\right), 1.35-2.05\left(\mathrm{~m}, 10 \mathrm{H},-\mathrm{OCH}_{2} \underline{\mathrm{C}}_{2} \mathrm{C}_{2^{-}},-\mathrm{CO}_{2} \mathrm{CH}_{2} \underline{\mathrm{C}}_{2}-\right), 2.36(\mathrm{t}, 2 \mathrm{H}$, $\left.-\underline{\mathrm{CH}}_{2} \mathrm{COOH}\right), 4.04\left(\mathrm{t}, 4 \mathrm{H}, \mathrm{ArOC} \underline{\mathrm{H}}_{2}-\right), 4.24\left(\mathrm{t}, 2 \mathrm{H},-\mathrm{CO}_{2} \underline{\mathrm{CH}}_{2}-\right), 6.98(\mathrm{dd}, 4 \mathrm{H}, \mathrm{ArH}), 7.26(\mathrm{~m}, 1 \mathrm{H}$, 
ArH), $7.46(\mathrm{dd}, 1 \mathrm{H}, \mathrm{ArH}), 7.90(\mathrm{~d}, 1 \mathrm{H}, \mathrm{ArH}), 8.15(\mathrm{dd}, 4 \mathrm{H}, \mathrm{ArH})$. FTIR (THF): $1739 \mathrm{~cm}^{-1}$ (vCO, ester, s), $1606 \mathrm{~cm}^{-1}$ (vCO, acid, s), $1511 \mathrm{~cm}^{-1}$ (vCO, acid, s).

6-(2,5-Di(4'-butyloxybenzoyloxy)benzoyloxy)hexanoic acid (3b). 3b was prepared by the same procedure as 3a by substituting 6-bromohexanoic acid for 4-bromobutyric acid. ${ }^{1} \mathrm{H}$ $\operatorname{NMR}\left(\mathrm{CDCl}_{3}\right): \delta 1.00\left(\mathrm{t}, 6 \mathrm{H},-\mathrm{CH}_{3}\right), 1.20-2.05\left(\mathrm{~m}, 14 \mathrm{H},-\mathrm{OCH}_{2} \underline{\mathrm{C}}_{2} \mathrm{C}_{2^{-}},-\mathrm{CO}_{2} \mathrm{CH}_{2}\left(\mathrm{C}_{2}\right)_{3^{-}}\right)$, $2.27\left(\mathrm{t}, 2 \mathrm{H},-\underline{\mathrm{C}}_{2} \mathrm{COOH}\right), 4.04\left(\mathrm{t}, 4 \mathrm{H}, \mathrm{ArOC}_{2_{2}}\right), 4.18\left(\mathrm{t}, 2 \mathrm{H},-\mathrm{CO}_{2} \underline{\mathrm{CH}}_{2}-\right), 6.98$ (dd, 4H, $\left.\mathrm{ArH}\right)$, $7.26(\mathrm{~m}, 1 \mathrm{H}, \operatorname{ArH}), 7.46(\mathrm{dd}, 1 \mathrm{H}, \operatorname{ArH}), 7.90(\mathrm{~d}, 1 \mathrm{H}, \operatorname{ArH}), 8.15(\mathrm{dd}, 4 \mathrm{H}, \operatorname{ArH})$. FTIR (THF): $1739 \mathrm{~cm}^{-1}$ (vCO, ester, s), $1606 \mathrm{~cm}^{-1}$ (vCO, acid, s), $1511 \mathrm{~cm}^{-1}$ (vCO, acid, s).

11-(2,5-Di(4'-butyloxybenzoyloxy)benzoyloxy)undecanoic acid (3c). 3c was prepared by the same procedure as 3a by substituting 11-bromoundecanoic acid for 4-bromobutyric acid. ${ }^{1} \mathrm{H}$ NMR (CDCl 3$): \delta 1.00$ (t, $\left.6 \mathrm{H},-\mathrm{CH}_{3}\right), 1.15-2.05\left(\mathrm{~m}, 24 \mathrm{H},-\mathrm{OCH}_{2} \mathrm{C}_{2} \mathrm{C}_{2} 2^{-},-\mathrm{CO}_{2} \mathrm{CH}_{2}\left(\mathrm{C}_{2}\right)_{8^{-}}\right)$, $2.34\left(\mathrm{t}, 2 \mathrm{H},-\mathrm{C}_{2} \mathrm{COOH}\right), 4.11\left(\mathrm{~m}, 6 \mathrm{H}, \mathrm{ArOC}_{\mathrm{H}_{2}},-\mathrm{CO}_{2} \underline{\mathrm{C}}_{2}{ }^{-}\right), 6.98(\mathrm{dd}, 4 \mathrm{H}, \mathrm{ArH}), 7.26(\mathrm{~m}, 1 \mathrm{H}$, ArH), $7.46(\mathrm{dd}, 1 \mathrm{H}, \mathrm{ArH}), 7.90(\mathrm{~d}, 1 \mathrm{H}, \mathrm{ArH}), 8.15(\mathrm{dd}, 4 \mathrm{H}, \mathrm{ArH})$. FTIR (THF): $1739 \mathrm{~cm}^{-1}$ (vCO, ester, s), $1606 \mathrm{~cm}^{-1}$ (vCO, acid, s), $1511 \mathrm{~cm}^{-1}$ (vCO, acid, s).

4-(2,5-Di(4'-butyloxybenzoyloxy)benzoyloxy)butyrate N-hydroxysuccinimidyl Ester (4a). Separate solutions of 3a (1.5 g, $2.5 \mathrm{mmol})$, N-hydroxysuccinimide (350 mg, $3.0 \mathrm{mmol})$ and $\mathrm{N}, \mathrm{N}^{\prime}$-dicyclohexylcarbodiimide $(550 \mathrm{mg}, 2.7 \mathrm{mmol})$ in $\mathrm{THF}(30 \mathrm{~mL})$ were cooled to $0{ }^{\circ} \mathrm{C}$, then mixed together and stirred for 30 minutes. The solution was then placed in a refrigerator $\left(4{ }^{\circ} \mathrm{C}\right)$ for 12 hours, after which insoluble N, N'-dicyclohexylurea was filtered off and the solvent was removed under vacuum. The residue was dissolved in ethyl acetate $(30 \mathrm{~mL})$ and washed with $\mathrm{H}_{2} \mathrm{O}(2 \times 30 \mathrm{~mL})$. The organic layer was dried over $\mathrm{MgSO}_{4}$ and the solvent evaporated. The residue was then recrystallized from THF, toluene and hexane (2:1:5) to give the product as a white solid $(1.5 \mathrm{~g}, 86 \%)$. ${ }^{1} \mathrm{H}$ NMR $\left(\mathrm{CDCl}_{3}\right): \delta 1.00\left(\mathrm{t}, 6 \mathrm{H},-\mathrm{CH}_{3}\right), 1.35-2.05(\mathrm{~m}, 10 \mathrm{H}$, - 
$\mathrm{OCH}_{2} \underline{\mathrm{C}}_{2} \mathrm{C}_{2^{-}},-\mathrm{CO}_{2} \mathrm{CH}_{2} \underline{\mathrm{C}}_{2^{-}}$), 2.48 (t, $2 \mathrm{H},-\mathrm{C}_{2} \mathrm{CO}_{2} \mathrm{~N}-$ ), 2.80 (s, $4 \mathrm{H}$, succinimide), 4.04 (t, 4H, $\left.\mathrm{ArOC}_{2} 2^{-}\right), 4.24\left(\mathrm{t}, 2 \mathrm{H},-\mathrm{CO}_{2} \underline{\mathrm{CH}}_{2}-\right), 6.98(\mathrm{dd}, 4 \mathrm{H}, \mathrm{ArH}), 7.26(\mathrm{~m}, 1 \mathrm{H}, \mathrm{ArH}), 7.46(\mathrm{dd}, 1 \mathrm{H}$, ArH), $7.90(\mathrm{~d}, 1 \mathrm{H}, \mathrm{ArH}), 8.15$ (dd, 4H, ArH). FTIR (THF): $1742 \mathrm{~cm}^{-1}$ (vCO, ester, s).

\section{6-(2,5-Di(4'-butyloxybenzoyloxy)benzoyloxy)hexanoate N-hydroxysuccinimidyl}

Ester (4a). 4b was prepared from $\mathbf{3 b}$ using the same procedure as with $4 \mathbf{a}$ ( $88 \%$ yield). ${ }^{1} \mathrm{H}$ $\operatorname{NMR}\left(\mathrm{CDCl}_{3}\right): \delta 1.00\left(\mathrm{t}, 6 \mathrm{H},-\mathrm{CH}_{3}\right), 1.20-2.05\left(\mathrm{~m}, 14 \mathrm{H},-\mathrm{OCH}_{2} \underline{\mathrm{C}}_{2} \mathrm{C}_{2^{-}},-\mathrm{CO}_{2} \mathrm{CH}_{2}\left(\mathrm{C}_{2}\right)_{3}{ }^{-}\right)$, $2.53\left(\mathrm{t}, 2 \mathrm{H},-\mathrm{CH}_{2} \mathrm{CO}_{2} \mathrm{~N}-\right), 2.81\left(\mathrm{~s}, 4 \mathrm{H}\right.$, succinimide), $4.04\left(\mathrm{t}, 4 \mathrm{H}, \mathrm{ArOC}_{2} 2^{-}\right), 4.18(\mathrm{t}, 2 \mathrm{H},-$ $\left.\mathrm{CO}_{2} \underline{\mathrm{C}}_{2^{-}}\right), 6.98$ (dd, 4H, ArH), $7.26(\mathrm{~m}, 1 \mathrm{H}, \mathrm{ArH}), 7.46$ (dd, 1H, ArH), 7.90 (d, 1H, ArH), 8.15 (dd, 4H, ArH). FTIR (THF): $1742 \mathrm{~cm}^{-1}$ (vCO, ester, s).

11-(2,5-Di(4'-butyloxybenzoyloxy)benzoyloxy)undecanoate N-hydroxysuccinimidyl

Ester (4a). $4 \mathbf{c}$ was prepared from $3 \mathbf{c}$ using the same procedure as with $4 \mathbf{a}\left(91 \%\right.$ yield). ${ }^{1} \mathrm{H}$ $\operatorname{NMR}\left(\mathrm{CDCl}_{3}\right): \delta 1.00\left(\mathrm{t}, 6 \mathrm{H},-\mathrm{CH}_{3}\right), 1.15-2.05\left(\mathrm{~m}, 24 \mathrm{H},-\mathrm{OCH}_{2} \underline{\mathrm{C}}_{2} \mathrm{C}_{2^{-}},-\mathrm{CO}_{2} \mathrm{CH}_{2}\left(\mathrm{C}_{2}\right)_{8^{-}}\right)$, $2.60\left(\mathrm{t}, 2 \mathrm{H},-\underline{\mathrm{C}}_{2} \mathrm{CO}_{2} \mathrm{~N}-\right), 2.83(\mathrm{~s}, 4 \mathrm{H}$, succinimide $) 4.11\left(\mathrm{~m}, 6 \mathrm{H}, \mathrm{ArOC}_{2^{-}},-\mathrm{CO}_{2} \underline{\mathrm{C}}_{2}-\right), 6.98$ (dd, 4H, ArH), 7.26 (m, 1H, ArH), 7.46 (dd, 1H, ArH), 7.90 (d, 1H, ArH), 8.15 (dd, 4H, ArH). FTIR (THF): $1742 \mathrm{~cm}^{-1}$ (vCO, ester, s).

$N_{\varepsilon^{-}}$(4-(2,5-Di(4'-butyloxybenzoyloxy)benzoyloxy)butanoyl)- $N_{\alpha^{-}}$-Z-L-Lysine (5a). $\mathrm{N}_{\alpha^{-}}$ Z-L-lysine (490 mg, $1.7 \mathrm{mmol})$ was suspended in THF $(50 \mathrm{~mL})$ and deionized water $(2 \mathrm{~mL})$ added to dissolve the solid. The solution was cooled to $0{ }^{\circ} \mathrm{C}$ and $4 \mathbf{a}(1.0 \mathrm{~g}, 1.5 \mathrm{mmol})$ in THF (5 $\mathrm{mL})$ at $0{ }^{\circ} \mathrm{C}$ and triethylamine $(0.3 \mathrm{~mL}, 2.25 \mathrm{mmol})$ were then added. The reaction was stirred for five minutes and then allowed to warm to room temperature and stirred for two days. The reaction was then quenched with glacial acetic acid $(2 \mathrm{~mL})$ and the volatiles were removed under vacuum. The residue was dissolved in ethyl acetate $(40 \mathrm{~mL})$, filtered, and washed with water $(2$ x $30 \mathrm{~mL}$ ). The organic layer was dried over $\mathrm{MgSO}_{4}$, evaporated, and the residue crystallized 
from THF, toluene and hexane $(2: 1: 5)$ to give the product as a white solid $(1.1 \mathrm{~g}, 74 \%) .{ }^{1} \mathrm{H}$ $\operatorname{NMR}\left(\mathrm{CDCl}_{3}\right): \delta 1.00\left(\mathrm{t}, 6 \mathrm{H},-\mathrm{CH}_{3}\right), 1.15-1.90\left(\mathrm{~m}, 16 \mathrm{H},-\mathrm{OCH}_{2} \underline{\mathrm{C}}_{2} \mathrm{C}_{2_{2}},-\mathrm{CONHCH}_{2}\left(\mathrm{C}_{2}\right)_{3^{-}},-\right.$ $\left.\mathrm{CO}_{2} \mathrm{CH}_{2} \mathrm{C}_{2}-\right), 2.07$ (t, $\left.2 \mathrm{H},-\mathrm{NC}(\mathrm{O}) \mathrm{C}_{2}-\right), 3.11\left(\mathrm{~m}, 2 \mathrm{H},-\underline{\mathrm{C}}_{2} \mathrm{NH}-\right), 4.05$ (t, 4H, $\left.\mathrm{ArOC} \underline{\mathrm{H}}_{2}-\right), 4.16$ $\left(\mathrm{t}, 2 \mathrm{H},-\mathrm{CO}_{2} \mathrm{C}_{2}-\right), 4.30(\mathrm{q}, 1 \mathrm{H}, \alpha-\mathrm{H}), 5.06\left(\mathrm{~s}, 2 \mathrm{H}, \mathrm{C}_{6} \mathrm{H}_{5} \mathrm{C}_{2}-\right), 5.71(\mathrm{~d}, 1 \mathrm{H},-\mathrm{C}(\mathrm{O}) \mathrm{N} \underline{\mathrm{H}}-), 5.98(\mathrm{t}$, 1H, -OC(O)NH-), 6.96 (dd, 4H, ArH), 7.25 (m, 5H, ArH), 7.43 (m, 1H, ArH), $7.86(\mathrm{~d}, 1 \mathrm{H}, \mathrm{ArH})$, 8.12 (dd, 4H, ArH). FTIR (THF): 1736, 1605, 1513, 1458, 1249, 1163, 1182, $910 \mathrm{~cm}^{-1}$.

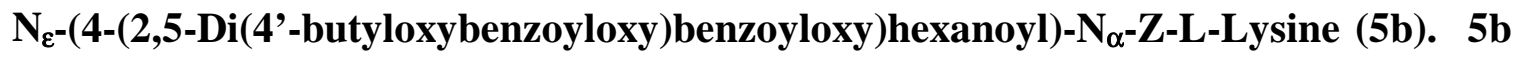

was prepared from $\mathbf{4 b}$ using the same procedure as with $\mathbf{5 a}\left(76 \%\right.$ yield). ${ }^{1} \mathrm{H}$ NMR $\left(\mathrm{CDCl}_{3}\right)$ : $\delta$ 1.00 (t, 6H, - $\left.\mathrm{CH}_{3}\right), 1.15-1.90\left(\mathrm{~m}, 20 \mathrm{H},-\mathrm{OCH}_{2} \mathrm{CH}_{2} \mathrm{CH}_{2^{-}},-\mathrm{CONHCH}_{2}\left(\mathrm{CH}_{2}\right)_{3^{-}},-\mathrm{CO}_{2} \mathrm{CH}_{2}\left(\mathrm{CH}_{2}\right)_{3^{-}}\right)$, 2.06 (t, $\left.2 \mathrm{H},-\mathrm{NC}(\mathrm{O}) \mathrm{C}_{2^{-}}\right), 3.15\left(\mathrm{~m}, 2 \mathrm{H},-\underline{\mathrm{C}}_{2} \mathrm{NH}-\right), 4.05$ (t, 4H, $\left.\mathrm{ArOC}_{2} 2^{-}\right), 4.14$ (t, $2 \mathrm{H},-$ $\left.\mathrm{CO}_{2} \underline{\mathrm{C}}_{2}-\right), 4.30(\mathrm{q}, 1 \mathrm{H}, \alpha-\mathrm{H}), 5.07\left(\mathrm{~s}, 2 \mathrm{H}, \mathrm{C}_{6} \mathrm{H}_{5} \mathrm{C}_{2^{-}}\right), 5.67(\mathrm{~d}, 1 \mathrm{H},-\mathrm{CON} \underline{\mathrm{H}}-), 5.88(\mathrm{t}, 1 \mathrm{H},-$ $\mathrm{OC}(\mathrm{O}) \mathrm{NH}-), 6.96$ (dd, 4H, ArH), 7.25 (m, 5H, ArH), 7.43 (m, 1H, ArH), 7.86 (d, 1H, ArH), 8.12 (dd, 4H, ArH). FTIR (THF): 1736, 1605, 1513, 1458, 1249, 1163, 1182, $910 \mathrm{~cm}^{-1}$.

$\mathrm{N}_{\varepsilon^{-}}$-(4-(2,5-Di(4'-butyloxybenzoyloxy)benzoyloxy)undecanoyl)- $\mathrm{N}_{\alpha}-\mathrm{Z}$-L-Lysine $\quad(5 \mathrm{c})$. 5c was prepared from $\mathbf{4 c}$ using the same procedure as with $\mathbf{5 a}\left(78 \%\right.$ yield). ${ }^{1} \mathrm{H} \mathrm{NMR}\left(\mathrm{CDCl}_{3}\right): \delta$ $1.00\left(\mathrm{t}, 6 \mathrm{H},-\mathrm{CH}_{3}\right), 1.15-1.90\left(\mathrm{~m}, 30 \mathrm{H},-\mathrm{OCH}_{2} \underline{\mathrm{C}}_{2} \mathrm{C}_{2} 2^{-},-\mathrm{CONHCH}_{2}\left(\mathrm{C}_{2}\right)_{3^{-}},-\mathrm{CO}_{2} \mathrm{CH}_{2}\left(\mathrm{CH}_{2}\right)_{8^{-}}\right)$, $2.06\left(\mathrm{t}, 2 \mathrm{H},-\mathrm{NC}(\mathrm{O}) \underline{\mathrm{C}}_{2}{ }^{-}\right), 3.15\left(\mathrm{~m}, 2 \mathrm{H},-\underline{\mathrm{C}}_{2} \mathrm{NH}-\right), 4.05\left(\mathrm{t}, 4 \mathrm{H}, \operatorname{ArOC} \underline{\mathrm{H}}_{2}-\right), 4.14(\mathrm{t}, 2 \mathrm{H},-$ $\left.\mathrm{CO}_{2} \underline{\mathrm{C}}_{2}{ }^{-}\right), 4.50(\mathrm{q}, 1 \mathrm{H}, \alpha-\mathrm{H}), 5.08\left(\mathrm{~s}, 2 \mathrm{H}, \mathrm{C}_{6} \mathrm{H}_{5} \underline{\mathrm{CH}}_{2}^{-}\right), 5.65$ (d, 1H, -CON$\left.\underline{\mathrm{H}}-\right), 5.78(\mathrm{t}, 1 \mathrm{H},-$

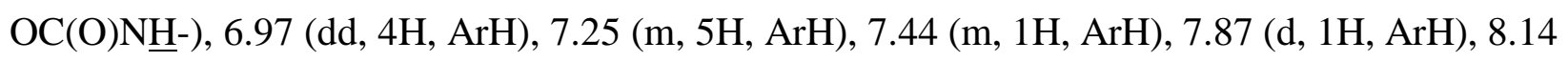
(dd, 4H, ArH). FTIR (THF): 1736, 1605, 1513, 1458, 1249, 1163, 1182, $910 \mathrm{~cm}^{-1}$.

\section{$\mathrm{N}_{\varepsilon}$-(4-(2,5-Di(4'-butyloxybenzoyloxy)benzoyloxy)butanoyl)-L-Lysine-N-}

carboxyanhydride (6a). To a solution of $\mathbf{5 a}(1.1 \mathrm{~g}, 1.3 \mathrm{mmol})$ in anhydrous $\mathrm{CH}_{2} \mathrm{Cl}_{2}(40 \mathrm{~mL})$ under dinitrogen in a Schlenk flask was added 1,1-dichlorodimethyl ether (0.12 mL, $1.5 \mathrm{mmol})$ 
via syringe. The solution was heated to reflux for three days under dinitrogen, after which the solvent was removed under vacuum. The residue was successively crystallized from a mixture of THF, toluene and hexane (3:1:6) three times in thea dry box to give the product as a white solid $(0.63 \mathrm{~g}, 65 \%) . \quad{ }^{1} \mathrm{H}$ NMR $\left(\mathrm{CDCl}_{3}\right): \delta 1.00\left(\mathrm{t}, 6 \mathrm{H},-\mathrm{CH}_{3}\right), 1.15-1.90(\mathrm{~m}, 16 \mathrm{H},-$ $\left.\mathrm{OCH}_{2} \underline{\mathrm{C}}_{2} \mathrm{C}_{2^{-}},-\mathrm{CONHCH}_{2}\left(\underline{\mathrm{C}}_{2}\right)_{3}-,-\mathrm{CO}_{2} \mathrm{CH}_{2} \underline{\mathrm{CH}}_{2}-\right), 2.07$ (t, $\left.2 \mathrm{H},-\mathrm{NC}(\mathrm{O}) \mathrm{C}_{2} 2^{-}\right), 3.11(\mathrm{~m}, 2 \mathrm{H},-$ $\left.\mathrm{C}_{2} \mathrm{NH}-\right), 4.05\left(\mathrm{t}, 4 \mathrm{H}, \mathrm{ArOC}_{2}-\right), 4.16\left(\mathrm{t}, 2 \mathrm{H},-\mathrm{CO}_{2} \underline{\mathrm{CH}}_{2}-\right), 4.30(\mathrm{q}, 1 \mathrm{H}, \alpha-\mathrm{H}), 5.71(\mathrm{~d}, 1 \mathrm{H},-$ $\mathrm{C}(\mathrm{O}) \mathrm{N} \underline{\mathrm{H}}-), 6.36(\mathrm{t}, 1 \mathrm{H},-\mathrm{O}(\mathrm{CO}) \mathrm{N} \underline{\mathrm{H}}-), 6.96(\mathrm{dd}, 4 \mathrm{H}, \mathrm{ArH}), 7.27(\mathrm{~m}, 1 \mathrm{H}, \mathrm{ArH}), 7.43(\mathrm{~m}, 1 \mathrm{H}$, ArH), $7.86(\mathrm{~d}, 1 \mathrm{H}, \operatorname{ArH}), 8.12(\mathrm{dd}, 4 \mathrm{H}, \operatorname{ArH})$. FTIR (THF): 1856, $1789 \mathrm{~cm}^{-1}(\mathrm{vCO}, \mathrm{NCA}, \mathrm{s})$, $1738 \mathrm{~cm}^{-1}(\mathrm{vCO}$, amide, $\mathrm{s})$.

$\mathrm{N}_{\varepsilon^{-}}$(4-(2,5-Di(4'-butyloxybenzoyloxy)benzoyloxy)hexanoyl)-L-Lysine-N-

carboxyanhydride (6b). $6 \mathbf{b}$ was prepared from $\mathbf{5 b}$ using the same procedure as with $\mathbf{6 a}(60 \%$ yield). ${ }^{1} \mathrm{H}$ NMR $\left(\mathrm{CDCl}_{3}\right): \delta 1.00\left(\mathrm{t}, 6 \mathrm{H},-\mathrm{CH}_{3}\right), 1.15-1.90\left(\mathrm{~m}, 20 \mathrm{H},-\mathrm{OCH}_{2} \mathrm{C}_{2} \mathrm{C}_{2} \underline{H}^{-},-\right.$ $\left.\mathrm{CONHCH}_{2}\left(\underline{\mathrm{C}}_{2}\right)_{3^{-}},-\mathrm{CO}_{2} \mathrm{CH}_{2}\left(\mathrm{C}_{2}\right)_{3^{-}}\right), 2.06\left(\mathrm{t}, 2 \mathrm{H},-\mathrm{NC}(\mathrm{O}) \mathrm{C}_{2}-\right), 3.15\left(\mathrm{~m}, 2 \mathrm{H},-\underline{\mathrm{H}}_{2} \mathrm{NH}-\right), 4.05$ (t, $\left.4 \mathrm{H}, \mathrm{ArOC}_{2}-\right), 4.14\left(\mathrm{t}, 2 \mathrm{H},-\mathrm{CO}_{2} \underline{\mathrm{C}}_{2}-\right), 4.30(\mathrm{q}, 1 \mathrm{H}, \alpha-\mathrm{H}), 5.67(\mathrm{~d}, 1 \mathrm{H},-\mathrm{C}(\mathrm{O}) \mathrm{N} \underline{\mathrm{H}}-), 6.41(\mathrm{t}$, 1H, $\left.-\mathrm{O}(\mathrm{CO}) \mathrm{NH}-{ }^{-}\right), 6.96(\mathrm{dd}, 4 \mathrm{H}, \mathrm{ArH}), 7.33(\mathrm{~m}, 1 \mathrm{H}, \mathrm{ArH}), 7.43(\mathrm{~m}, 1 \mathrm{H}, \mathrm{ArH}), 7.86(\mathrm{~d}, 1 \mathrm{H}, \mathrm{ArH})$ 8.12 (dd, 4H, ArH). FTIR (THF): 1855, $1789 \mathrm{~cm}^{-1}(\mathrm{vCO}, \mathrm{NCA}, \mathrm{s}), 1737 \mathrm{~cm}^{-1}(\mathrm{vCO}$, amide, s).

$\mathrm{N}_{\varepsilon^{-}}$(4-(2,5-Di(4'-butyloxybenzoyloxy)benzoyloxy)undecanoyl)-L-Lysine-N-

carboxyanhydride (6c). $6 \mathbf{c}$ was prepared from $\mathbf{5 c}$ using the same procedure as with $\mathbf{6 a}$ (64\% yield). ${ }^{1} \mathrm{H}$ NMR $\left(\mathrm{CDCl}_{3}\right): \delta 1.00\left(\mathrm{t}, 6 \mathrm{H},-\mathrm{CH}_{3}\right), 1.15-1.90\left(\mathrm{~m}, 30 \mathrm{H},-\mathrm{OCH}_{2} \mathrm{C}_{2} \mathrm{C}_{2} \underline{H}^{-},-\right.$ $\left.\mathrm{CONHCH}_{2}\left(\mathrm{C}_{2}\right)_{3^{-}},-\mathrm{CO}_{2} \mathrm{CH}_{2}\left(\underline{\mathrm{CH}}_{2}\right)_{8^{-}}\right), 2.06\left(\mathrm{t}, 2 \mathrm{H},-\mathrm{NC}(\mathrm{O}) \mathrm{C}_{2}{ }^{-}\right), 3.15\left(\mathrm{~m}, 2 \mathrm{H},-\underline{\mathrm{H}}_{2} \mathrm{NH}-\right), 4.05$ $\left(\mathrm{t}, 4 \mathrm{H}, \mathrm{ArOC}_{2}-\right), 4.14\left(\mathrm{t}, 2 \mathrm{H},-\mathrm{CO}_{2} \underline{\mathrm{C}}_{2}-\right), 4.50(\mathrm{q}, 1 \mathrm{H}, \alpha-\mathrm{H}), 5.65(\mathrm{~d}, 1 \mathrm{H},-\mathrm{C}(\mathrm{O}) \mathrm{N} \underline{\mathrm{H}}-), 6.38(\mathrm{t}$, 1H, -OC(O)N $\underline{\mathrm{H}-}), 6.97$ (dd, 4H, ArH), $7.34(\mathrm{~m}, 1 \mathrm{H}, \mathrm{ArH}), 7.44(\mathrm{~m}, 1 \mathrm{H}, \mathrm{ArH}), 7.87(\mathrm{~d}, 1 \mathrm{H}, \mathrm{ArH})$, 8.14 (dd, 4H, ArH). FTIR (THF): 1855, $1789 \mathrm{~cm}^{-1}(\mathrm{vCO}, \mathrm{NCA}, \mathrm{s}), 1740 \mathrm{~cm}^{-1}$ (vCO, amide, s). 


\section{$\operatorname{Poly}\left(\mathbf{N}_{\varepsilon^{-}}\right.$(4-(2,5-Di(4'-butyloxybenzoyloxy)benzoyloxy)butanoyl)-L-Lysine (7a). In}

the dry box $6 \mathbf{a}(100 \mathrm{mg}, 130 \mu \mathrm{mol})$ was dissolved in THF $(5 \mathrm{~mL})$ in a $25 \mathrm{~mL}$ reaction tube with a Teflon cap. An aliquot of $\left(\mathrm{PMe}_{3}\right)_{4} \mathrm{Co}(50 \mu \mathrm{L}$ of a $40 \mathrm{mM}$ solution in THF) was then injected into the NCA solution followed by capping of the flask and vigorous stirring. After ca. 45 minutes, an aliquot was removed to ensure complete consumption of NCA monomer by FTIR analysis of the monomer absorptions at 1855 and $1789 \mathrm{~cm}^{-1}$. When these absorptions were no longer present, the reaction mixture was transferred out of the dry box and polymer was precipitated by addition of methanol $(25 \mathrm{~mL})$. The solid was washed with methanol $(2 \times 25 \mathrm{~mL})$ and then dried in vacuo to yield the polymer as a white fibrous solid (92 mg, $98 \%$ ). FTIR (THF): $1737 \mathrm{~cm}^{-1}$ (vCO, ester, s), $1651 \mathrm{~cm}^{-1}$ (Amide-I, s), $1543 \mathrm{~cm}^{-1}$ (Amide-II, s). GPC (THF): $\mathrm{M}_{\mathrm{n}}=20,630 ; \mathrm{M}_{\mathrm{w}}$ $=39,710 ; \mathrm{M}_{\mathrm{w}} / \mathrm{M}_{\mathrm{n}}=1.93$.

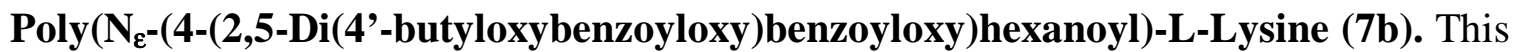
polymer was prepared from $\mathbf{6 b}$ using the same procedure as with $6 \mathbf{a}$ (96\% yield). FTIR (THF): $1737 \mathrm{~cm}^{-1}$ (vCO, ester, s), $1651 \mathrm{~cm}^{-1}$ (Amide-I, s), $1543 \mathrm{~cm}^{-1}$ (Amide-II, s). GPC (THF): $\mathrm{M}_{\mathrm{n}}=$ 13,$150 ; \mathrm{M}_{\mathrm{w}}=19,430 ; \mathrm{M}_{\mathrm{w}} / \mathrm{M}_{\mathrm{n}}=1.48$.

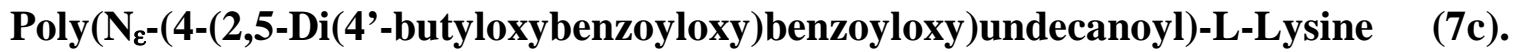
This polymer was prepared from 6c using the same procedure as with 6a (94\% yield). FTIR (THF): $1737 \mathrm{~cm}^{-1}$ (vCO, ester, s), $1651 \mathrm{~cm}^{-1}$ (Amide-I, s), $1543 \mathrm{~cm}^{-1}$ (Amide-II, s). GPC (THF): $\mathrm{M}_{\mathrm{n}}=22,370 ; \mathrm{M}_{\mathrm{w}}=43,360 ; \mathrm{M}_{\mathrm{w}} / \mathrm{M}_{\mathrm{n}}=1.94$ 


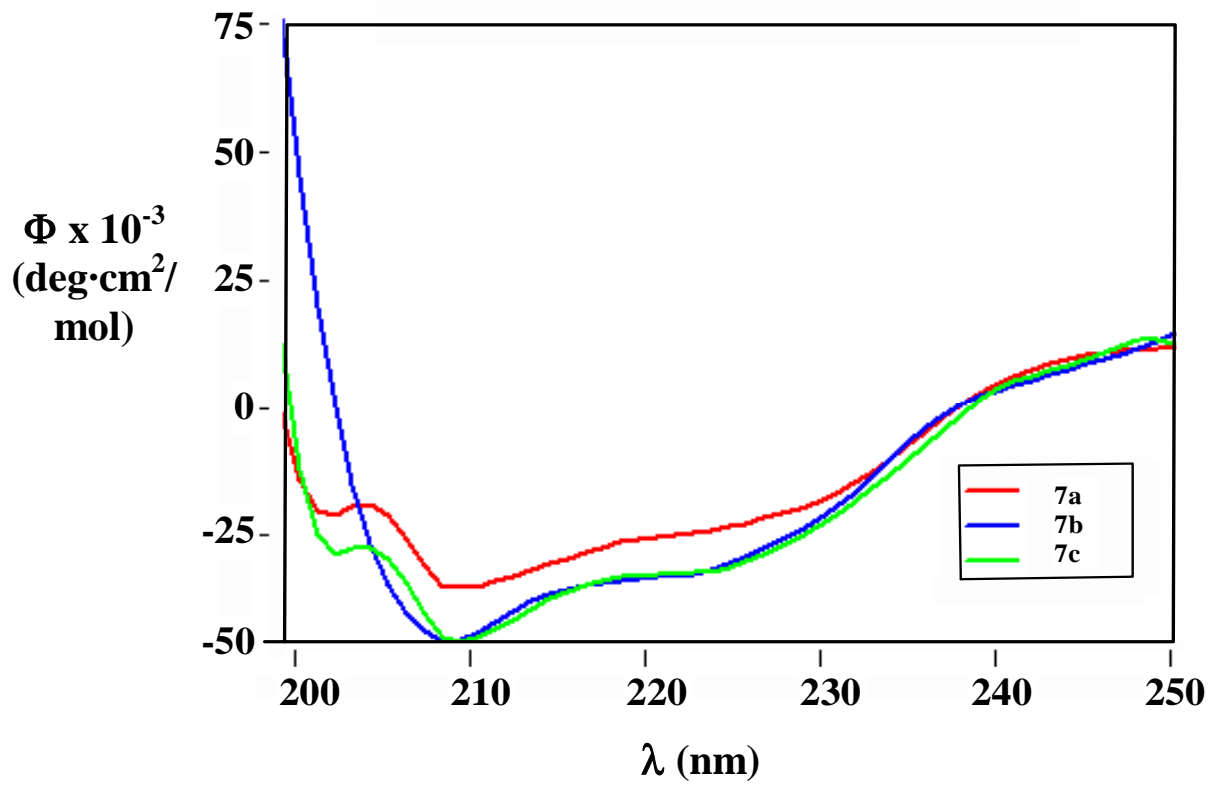

Figure S3 CD spectra of polypeptides 7a,b,c. All spectra show minima at 208 and 222 $\mathrm{nm}$, characteristic of an $\alpha$-helix structure. However, contributions from the aromatic sidechains prohibit quantitative analysis of the spectra. 